INPLASY

PROTOCOL

To cite: Oliveira et al. Effects of antibiotic-loaded chitosan nanoparticles against resistant bacteria: a systematic review. Inplasy protocol 202160069. doi:

10.37766/inplasy2021.6.0069

Received: 20 June 2021

Published: 20 June 2021

Corresponding author: Mariana Oliveira

dra.marisimoes@gmail.com

Author Affiliation:

Universidade Federal de Juiz de Fora (UFJF).

Support: UFJF.

Review Stage at time of this submission: The review has not yet started.

Conflicts of interest: None declared.

\section{Effects of antibiotic-loaded chitosan nanoparticles against resistant bacteria: a systematic review}

Oliveira, MS1; Souza, VGC2; Tavares, GD3; Fabri, RL4; Apolônio, $\mathrm{ACM}^{5}$.

Review question / Objective: Are antimicrobials encapsulated in chitosan nanoparticles more effective for resistant bacteria?

Condition being studied: For many years, antimicrobial agents have been used to inhibit or kill bacteria and other microorganisms. However, microbial resistance to these agents has developed on a very large scale over time, greatly reducing their effectiveness. Drug-resistant bacterial infections result in higher drug doses, addition of treatments with greater toxicity, longer hospital stays, and increased mortality. One of the most promising strategies to overcome microbial resistance is the use of nanoparticles. Several types of nanoparticles prevent the development of resistance, simultaneously using different mechanisms to fight microorganisms. Among these nanomaterials, chitosan nanoparticles can be highlighted, due to their various applications, mainly as a drug delivery system. Thanks to its bioadhesive properties, there may be an increase in the permanence time of chitosan systems in specific absorption sites, releasing the drug in a controlled manner and improving its bioavailability. So is it possible that antimicrobials encapsulated in chitosan particles are more effective than classic antimicrobials against resistant bacteria?

INPLASY registration number: This protocol was registered with the International Platform of Registered Systematic Review and Meta-Analysis Protocols (INPLASY) on 20 June 2021 and was last updated on 20 June 2021 (registration number INPLASY202160069).

\section{INTRODUCTION}

Review question / Objective: Are antimicrobials encapsulated in chitosan nanoparticles more effective for resistant bacteria?

Condition being studied: For many years, antimicrobial agents have been used to 
inhibit or kill bacteria and other microorganisms. However, microbial resistance to these agents has developed on a very large scale over time, greatly reducing their effectiveness. Drug-resistant bacterial infections result in higher drug doses, addition of treatments with greater toxicity, longer hospital stays, and increased mortality. One of the most promising strategies to overcome microbial resistance is the use of nanoparticles. Several types of nanoparticles prevent the development of resistance, simultaneously using different mechanisms to fight microorganisms. Among these nanomaterials, chitosan nanoparticles can be highlighted, due to their various applications, mainly as a drug delivery system. Thanks to its bioadhesive properties, there may be an increase in the permanence time of chitosan systems in specific absorption sites, releasing the drug in a controlled manner and improving its bioavailability. So is it possible that antimicrobials encapsulated in chitosan particles are more effective than classic antimicrobials against resistant bacteria?

\section{METHODS}

Search strategy: An electronic search will be conducted through MEDLINE/PubMed, Web of Science, Scopus, BVS, EMBASE and MEDLINE/Ovid databases from inception to July 2021. The search will be conducted using the following Medical Subject Headings (MeSH) descriptors and key words in combination with the Boolean operators "AND" and "OR": "chitosan", "nanoparticles", "nanomaterials", "AntiInfective Agents", "Anti Bacterial Agents", "Antibacterial Agent", "antibiotic", "antimicrobial agents", "Drug resistance, bacterial", "Drug resistance", "bacterial resistance", "multidrug resistance", "antimicrobial resistance".

Participant or population: Drug resistant bacteria.

Intervention: Loaded chitosan nanoparticles.

Comparator: classic antimicrobial agent.
Study designs to be included: in vitro and in vivo studies.

Eligibility criteria: Studies with antibioticloaded chitosan nanoparticles, in English, Portuguese or Spanish, without date restriction.

Information sources: At first, the search will be conducted with electronic databases, then, additional literature search will be performed from the reference list of identified eligible studies. Authors will be contacted to obtain missing information from the studies.

Main outcome(s): We believe our findings will confirm chitosan's antimicrobial properties, as well as its ability to carry and enhance the effect of classic antimicrobials, thus combating drug resistance. For all the research, a year will be necessary.

Data management: For the identification of studies included or considered for this review, detailed search strategies will be developed for each database searched. These will be based on the search strategy developed for PubMed and adapted appropriately for each database to take account of differences in controlled vocabulary and syntax rules. The titles and abstracts of the studies that will be obtained from the research will be independently selected by two reviewers to select studies that potentially met the inclusion criteria. Based on the summary, full text versions of potentially relevant articles will be obtained. Disagreements concerning eligibility will be resolved by consensus and, if the disagreement persists, the decision will be settled by arbitration by a third reviewer. Articles that meet all inclusion criteria will be processed for data extraction. A PRISMA (Preferred Reporting Items for Systematic Reviews and Meta-Analyses) flow chart will be compiled to summarize the study selection process. The characteristics of the population, intervention, comparison and results will be extracted independently of all studies by a reviewer with a specially designed form of data extraction. A second 
reviewer re-reads the full texts of the included studies and independently of the other verifies the extracted data. Disagreement between reviewers will be resolved through discussion and consensus. If this is not satisfactory, the judgment of a third reviewer is decisive.

Quality assessment / Risk of bias analysis: A specially designed and standardized data-extraction table will be used to extract data from the included studies for assessment of study quality and evidence synthesis. All included studies will be independently scored for their methodological quality by two reviewers. Disagreement between the reviewers will be resolved through discussion and consensus. If this is not satisfactory, the judgement of a third reviewer is decisive.

Strategy of data synthesis: Subgroups considering the country of the study conducted; sample size; type of study, bacteria and antibiotic agent used; outcome will be performed.

Subgroup analysis: A descriptive presentation of the data will be used for all studies.

Sensitivity analysis: Subsequently, if possible, a meta-analysis (MA) will be performed.

Country(ies) involved: Brazil.

Keywords: chitosan; nanoparticles; nanomaterials; drug resistance; antibacterial agents.

Contributions of each author:

Author 1 - Mariana Oliveira.

Author 2 - Vívian Souza.

Author 3 - Guilherme Tavares.

Author 4 - Rodrigo Fabri.

Author 5 - Ana Carolina Apolônio. 